\title{
A Role for Prefrontal Cortex in Memory Storage for Trace Fear Conditioning
}

\author{
Jason D. Runyan, Anthony N. Moore, and Pramod K. Dash \\ The Vivian L. Smith Center for Neurologic Research and the Department of Neurobiology and Anatomy, The University of Texas Medical School, Houston, \\ Texas 77225
}

\begin{abstract}
The prefrontal cortex has been shown to participate in the association of events separated by time. However, it is not known whether the prefrontal cortex stores the memory for these relationships. Trace conditioning is a form of classical conditioning in which a time gap separates the conditioned stimulus (CS) from the unconditioned stimulus (US), the association of which has been shown to depend on prefrontal activity. Here we demonstrate that inhibition of extracellular signal-regulated kinase (Erk) cascade (a biochemical pathway involved in long-term memory storage) in the rat medial prefrontal cortex did not interfere with memory encoding for trace fear conditioning but impaired memory retention. In addition, animals displayed impaired memory for the irrelevancy of the training context. Hippocampal Erk phosphorylation was found to have a later time course than prefrontal Erk phosphorylation after trace fear conditioning, indicating a direct role for the prefrontal cortex in associative memory storage for temporally separated events as well as in memory storage of relevancy.
\end{abstract}

Key words: prefrontal cortex; memory storage; trace conditioning; Erk/MAPK; relevancy; hippocampus

\section{Introduction}

The prefrontal cortex plays a critical role for behaviors that require a high level of mental integration by regulating selection (Dias et al., 1996), representation (Fuster and Alexander, 1971), monitoring (Everling et al., 2002), and interpretation (Dolan and Fletcher, 1997; Schoenbaum et al., 1998) of multimodal stimuli. In addition, the prefrontal cortex participates in the association of temporally separated events (Fuster et al., 2000), with damage to this structure resulting in an inability to select, maintain, and associate temporally disconnected stimuli (Dias et al., 1997). Patients with frontal lobe damage often display memory deficits (Markowitsch et al., 1993) as well as socially inappropriate behaviors (Harlow, 1848; Morris et al., 1997). Although these dysfunctions have been attributed to disruptions in prefrontal activity and an inability to learn, they also could, however, result from a deficit in prefrontal memory storage.

Trace conditioning (Pavlov, 1927) is a form of learning that requires the association of a conditioned stimulus (CS) and an unconditioned stimulus (US) separated by time. Human studies have indicated that trace conditioning is a declarative task that involves the hippocampus (Clark and Squire, 1998). Additionally, animal studies have shown that medial prefrontal cortical (mPFC; prelimbic/infralimbic cortices) lesions result in impaired trace eye-blink conditioning, but not delayed conditioning

Received 0ct. 30, 2003; revised Dec. 6, 2003; accepted Dec. 10, 2003.

This research was supported by National Institutes of Health Grant NS 35457 . We thank Melanie Moody and Sara Mach for technical assistance and Dr. Raymond Grill for help with the confocal imagery.

Correspondence should be addressed to Pramod Dash, Department of Neurobiology, The University of Texas Medical School, P.0. Box 20708, Houston, TX 77225. E-mail: p.dash@uth.tmc.edu. DOI:10.1523/JNEUROSCI.4880-03.2004

Copyright $\odot 2004$ Society for Neuroscience $\quad$ 0270-6474/04/241288-08\$15.00/0
(Kronforst-Collins and Disterhoft, 1998; McLaughlin et al., 2002). Although studies have designated a prefrontal involvement in trace conditioning, it has not been determined whether this includes memory storage.

The phosphorylation and activation of the extracellular signal-regulated kinase (Erk) cascade have been shown to be required for long-term, but not short-term, memory in several organisms including invertebrates (Martin et al., 1997; Crow et al., 1998) and vertebrates (Atkins et al., 1998, Blum et al., 1999). In mammals the activation and necessity of Erk within structures including the insular cortex (Berman et al., 1998), the hippocampus (Blum et al., 1999), the entorhinal cortex (Hebert and Dash, 2002), and the amygdala (Schafe et al., 2000) have been demonstrated to be required in various memory tasks. Unlike lesion studies, inhibition of Erk allows for the assessment of long-term memory storage within a structure without causing a generalized dysfunction evidenced by intact acquisition (Blum et al., 1999) and short-term memory (Martin et al., 1997; Schafe et al., 2000). Taken together, these and other studies indicate that the phosphorylation and activation of Erk are a molecular correlate of long-term memory storage and that its inhibition can be used as a means of determining whether long-term memory occurs within a structure engaged in the learning event.

In this report we examine the role of the $\mathrm{mPFC}$ in storing long-term memory for trace fear conditioning by assessing the phosphorylation of Erk and determining the consequences of its inhibition. We present data to show that, in addition to its well established role in memory encoding and retrieval, long-term storage of information associating temporally disconnected events, as well as for the relevancy of stored associations, occurs in the $\mathrm{mPFC}$ in parallel with memory storage in the hippocampus. 


\section{Materials and Methods}

Subjects. Male Sprague Dawley rats (Charles River Laboratories, Wilmington, MA) weighing 250-300 gm were pair-housed under temperature-controlled conditions with a $12 \mathrm{hr}$ light/dark cycle. All rats were given ad libitum access to water and food. Animal protocols were approved by the institutional animal welfare committee and were in compliance with National Institutes of Health Guide for Care and Use of Laboratory Animals.

Surgery. Rats initially were anesthetized by using $5 \%$ isoflurane with a $2: 1 \mathrm{~N}_{2} \mathrm{O} / \mathrm{O}_{2}$ mixture and then were maintained with a $2.5 \%$ isoflurane/ 2:1 $\mathrm{N}_{2} \mathrm{O} / \mathrm{O}_{2}$ mixture via a face mask. Stainless steel guide cannulas (22 gauge) were implanted into the prelimbic area (bregma $+3.2 \mathrm{~mm}$, lateral $\pm 0.75 \mathrm{~mm}$, and depth $-2.5 \mathrm{~mm}$ ) or into the hippocampus (bregma $-3.3 \mathrm{~mm}$, lateral $\pm 2 \mathrm{~mm}$, and depth $-2.5 \mathrm{~mm}$ ) with a stereotaxic device. Dummy cannulas were placed in the guide cannulas during nonuse. Animals were given a $10 \mathrm{~d}$ rest period after surgery before behavioral testing. Stylettes that extended $1.5 \mathrm{~mm}$ past the end of the cannula were used for drug infusions.

Pharmacological infusions. U0126 was used as a selective mitogenactivated protein (MAP)/Erk kinase (MEK) inhibitor (Roberson et al., 1999; Davies et al., 2000). U0126 (Sigma, St. Louis, MO) was dissolved in a $35 \%$ DMSO and saline solution. Then $1.0 \mu \mathrm{g} / 0.5 \mu \mathrm{l}$ of U0126 was infused bilaterally into the prelimbic/infralimbic cortices at a rate of 0.25 $\mu \mathrm{l} / \mathrm{min}$ by a motorized microinfusion pump (Stoelting, Wood Dale, IL) $10 \mathrm{~min}$ before behavioral training in the pretraining infusion and immediately after training in the post-training experiment. The same protocol was followed for hippocampal post-training infusions performed at 15 min after training. All vehicle control infusions consisted of the same volume of solution used for the pharmacological inhibitor.

Unilateral intra-prelimbic/infralimbic infusions of U0126 (1.0 $\mu \mathrm{g} / 0.5$ $\mu \mathrm{l})$ were performed to test the effectiveness of the drug in inhibiting medial prefrontal Erk phosphorylation. The contralateral hemisphere of the same animal was infused with vehicle composed of the same percentage of DMSO in saline solution (35\% DMSO). All infusions were performed in freely moving animals. After all infusions the needles were left in place for $2 \mathrm{~min}$ to allow for diffusion of the drug, and sterilized dummy cannulas were replaced. Animals were allowed to recover from the infusion in their home cages.

Behaviorial procedure. All behavioral tests were performed by an investigator who was blind to the treatment groups. Animals were placed in the training context (Habitest Unit, Coulbourn Instruments, Allentown, $\mathrm{PA}$ ) and given a $120 \mathrm{sec}$ habituation period. Conditioning trials began with a $10 \mathrm{sec}$ tone (CS), followed by a $20 \mathrm{sec}$ trace period before the animal received a $0.7 \mathrm{sec}(0.8 \mathrm{~mA})$ foot shock (US). Freezing behavior was measured every $2 \mathrm{sec}$ during the trace period. Freezing behavior is defined as the absence of all movement, excluding movement caused by respiration. The conditioning trial was followed by a pseudo-random intertrial interval (ITI) that varied between 1 and $4 \mathrm{~min}$. This was so that amount of time between shocks could not be used as a cue for foot-shock onset. Conditioning trials were repeated seven times during an $18 \mathrm{~min}$ training period to maximize CS-US association and minimize context-US association because of the length of time in the context without receiving the US. The training protocol was optimized to allow for three main objectives: (1) to observe a gradual acquisition curve, (2) to maximize CS-US association and minimize context-US association, and (3) to allow for long-term retention at 48 and $72 \mathrm{hr}$. Similar trace fear conditioning protocols, with comparable trace periods, have been shown to require the hippocampus (Quinn et al., 2002).

During retention testing the animal was placed in a novel context and given a $120 \mathrm{sec}$ habituation period. In the absence of foot shock a $10 \mathrm{sec}$ tone was given, followed by a varied ITI period. This protocol was repeated a total of four times for post-training infusions and three times in pretraining infusion studies. Only three retention trials were used in pretraining infusion studies because of the lower overall retention observed in both vehicle and drug-infused animals after pretraining infusions. The percentage of freezing was measured every $2 \mathrm{sec}$ during the time of the trace period $(20 \mathrm{sec})$ after the tone. Freezing was used as a behavioral means to score the animals' ability to retain the CS-US rela- tionship or the contextual-US relationship. Contextual retention was measured $72 \mathrm{hr}$ after training by scoring the animals' freezing behavior when placed back into the original training context for a $50 \mathrm{sec}$ period without receiving the CS or US. For retraining experiments the animals first were trained in the trace conditioning task and then infused with U0126 $(1 \mu \mathrm{g})$ or vehicle 1 week later to avoid any effect of U0126 infusion on the previous training memory. At $48 \mathrm{hr}$ after these infusions the animals were retrained with the same trace behavioral protocol used in the training. Retention of CS-US association and context-US association was measured at $48 \mathrm{hr}$ by monitoring freezing behavior.

US-alone, CS-alone, delayed conditioning, and unpaired behavioral procedures were used for experimental control groups. The US-alone group was exposed to the same protocol as the trace fear conditioning group minus the foot shock (US). The CS-alone group was exposed to the same protocol as the trace fear conditioning group minus the tone (CS). Delay conditioning consisted of three $10 \mathrm{sec}$ tone and $0.7 \mathrm{sec}(0.8 \mathrm{~mA})$ foot-shock pairings in which the tone and foot shock coterminated, separated by a $64 \mathrm{sec}$ ITI. This behavioral protocol has been shown to elicit long-term retention of CS-US association (Dash et al., 2002). Unpaired trials consisted of the same number of tone and foot-shock exposures as the trace fear conditioning group within the same time frame. However, in the unpaired trials the tone and the foot shock were presented in a pseudo-random manner so that the offset of the tone could not be used as a predictor of the foot-shock onset. This was achieved by varying the order as well as the time interval separating the two stimuli.

Immunohistochemistry. Immediately and at 1 and $4 \mathrm{hr}$ after trace fear conditioning the prefrontal tissue from the left hemisphere was cut from the brain under ice-cold conditions in artificial CSF [ACSF; containing (in mM): 10 HEPES, $\mathrm{pH} 7.2,1.3 \mathrm{NaH}_{2} \mathrm{PO}_{4}, 3 \mathrm{KCl}, 124 \mathrm{NaCl}, 10$ dextrose, $26 \mathrm{NaHCO}_{3}$, and $2 \mathrm{MgCl}_{2}$ ] containing phosphatase inhibitors [containing (in mM) $2 \mathrm{NaF}, 2 \mathrm{Na}_{2} \mathrm{MoO}_{4}, 1 \mathrm{Na}_{3} \mathrm{VO}_{4}$ ]. The corpus callosum was used as a landmark to locate the medial prefrontal cortex. The same preparation protocol was used for all controls. Hippocampal preparations were performed from the same animals and in the same manner as medial prefrontal preparations. All tissue was fixed for $24 \mathrm{hr}$ with ice-cold $4 \%$ paraformaldehyde $/ 15 \%$ picric acid and then cryoprotected for $24 \mathrm{hr}$ in a $30 \%$ sucrose solution in PBS. Then $40 \mu \mathrm{m}$ sections were sliced with a cryostat and stored in tissue-collecting solution (24\% glycerin, $28.5 \%$ ethylene glycol, $47.5 \% \mathrm{PBS}$ ) at $-20^{\circ} \mathrm{C}$. For immunohistochemistry the slices were preblocked in a $5 \%$ BSA, $1 \times$ PBS solution for $2 \mathrm{hr}$. Freefloating sections were incubated overnight with a primary antibody in a $2.5 \%$ BSA, $1 \times$ PBS solution containing $0.1 \%$ Triton X-100. Slices were washed three times in a $1 \times$ PBS solution. Binding of the phospho-Erk ( 1 $\mu \mathrm{g} / \mathrm{ml}$ ) or phospho-CaMKII Thr ${ }^{286}(2 \mu \mathrm{g} / \mathrm{ml}$ ) antibodies (Cell Signaling Technology, Beverly, MA) were visualized by using species-specific secondary antibodies conjugated to an Alexa 568 fluorochrome $(2 \mu \mathrm{g} / \mathrm{ml}$; Molecular Probes, Eugene, OR). For double-labeled immunohistochemistry, phospho-Erk was detected as described above. NeuN $(1 \mu \mathrm{g} / \mathrm{ml})$, Rip $(1 \mu \mathrm{g} / \mathrm{ml})$, or GFAP $(2 \mu \mathrm{g} / \mathrm{ml})$ antibody was visualized by using anti-mouse secondary antibodies conjugated to an Alexa 488 fluorochrome $(2 \mu \mathrm{g} / \mathrm{ml})$ for confocal and additional imaging. Slices were incubated for $1.5 \mathrm{hr}$ in the secondary antibody.

Quantification of phospho-Erk-labeled cells. Comparable frontal sections ( $\sim 3.2 \mathrm{~mm}$ anterior to bregma) were selected for cell counts by the optical dissector methodology (Coggeshall and Lekan, 1996). Every third section was stained for phospho-Erk as described in Immunohistochemistry. Instead of fluorescence, DAB-ABC (Vector Laboratories, Burlingame, CA) staining was used to label phospho-Erk for counting purposes. Cells were quantified within a defined boundary of $2.15 \times 10^{-2}$ $\mathrm{mm}^{3}$ encompassing the prelimbic cortex, using a stereological investigator program, Stereo Investigator, Optical Dissector Probe (MicroBrightField, Colchester, VT) at $40 \times$ magnification. The results obtained from four independent sections per animal were averaged. The protocol used for quantification of phospho-Erk labeled cells within the prelimbic area was similar to previously established stereological protocols (Vann et al., 2000). Cell counts within specific prelimbic cortical layers were performed by defining each layer on the basis of NeuN immunoreactivity. Cell counts were performed by two blind independent researchers. The 


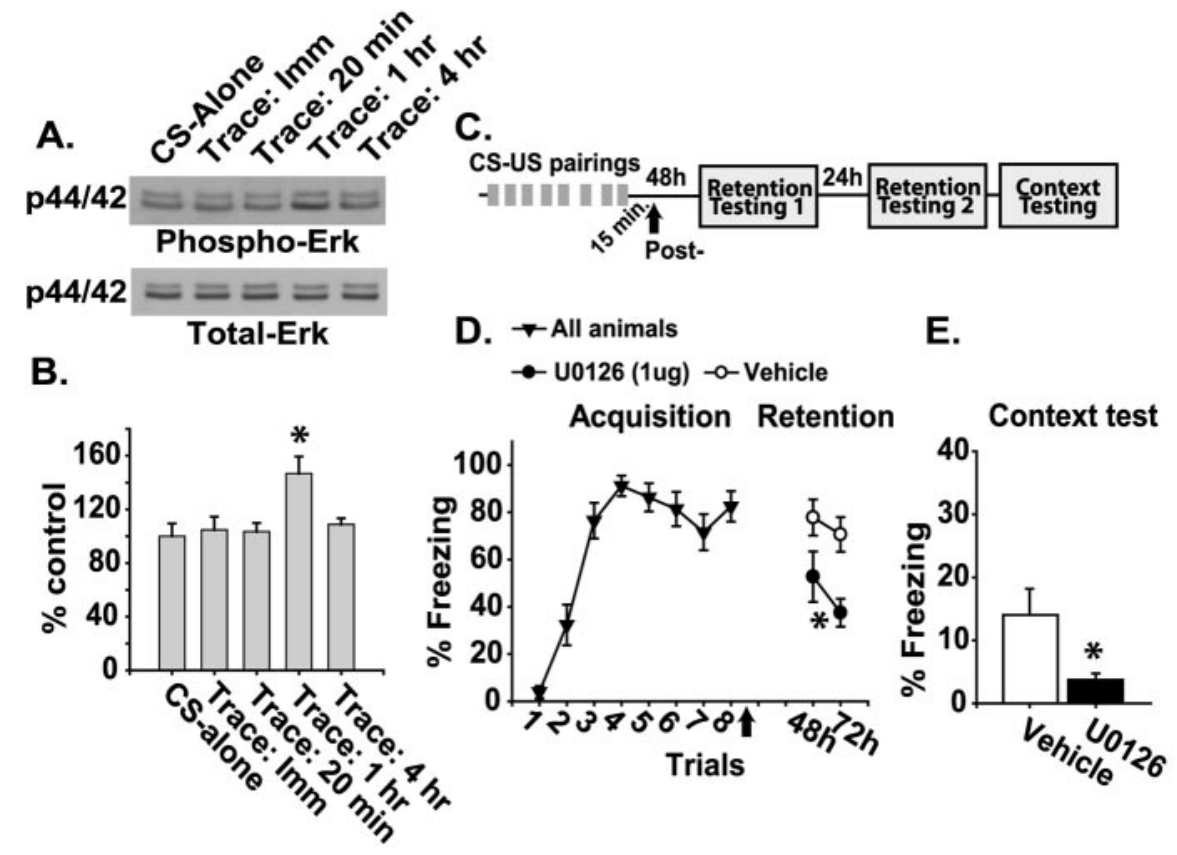

Figure 1. Hippocampal Erk phosphorylation as a correlate for long-term memory storage in trace conditioning. A, Representative Western blots for dually phosphorylated Erk (p44/42) and total Erk in samples taken from the hippocampus after the CS-alone control or immediately, $20 \mathrm{~min}$, or 1 or $4 \mathrm{hr}$ after trace fear conditioning. $B$, Graph of the mean \pm SEM for phospho-Erk immunoreactivity, normalized by using the total Erk immunoreactivity of the same blots for each condition. C, Illustration of the sequence of trace-conditioning training trials (CS-US pairings), infusion, and retention testing. D, Graph of the mean \pm SEM freezing behavior during the trace period after $C S$ offset, expressed as a percentage. Intrahippocampal U0126 infusion was administered 15 min post-training (indicated by the arrow) during (S-US retention testing. E, Graph of the mean \pm SEM freezing behavior expressed as a percentage to the training context. Asterisks indicate a significant difference as compared with control.

corpus callosum was used as a marker to locate the medial prefrontal cortex.

Western immunoblot analysis. At the appropriate time points after behavioral training the animals were decapitated. The prelimbic/infralimbic cortices $\left(\sim 1 \mathrm{~mm}^{3}\right.$ punch $)$ and the hippocampi were dissected in $1 \times$ ACSF with phosphatase inhibitors under ice-cold conditions. Cortical and hippocampal tissues were homogenized separately with a motorized glass Teflon homogenizer in a buffer containing (in mM): 10 Tris- $\mathrm{HCl}$, pH 7.4, 1 EGTA, 1 EDTA, 0.5 DTT, 0.5 PMSF, $2 \mathrm{NaF}, 2 \mathrm{Na}_{2} \mathrm{MoO}_{4}$, and 1 $\mathrm{Na}_{3} \mathrm{VO}_{4}$ plus $0.1 \mu \mathrm{M}$ okadaic acid and $10 \mu \mathrm{g} / \mathrm{ml}$ leupeptin and phosphatase inhibitors. These samples were stored at $-80^{\circ} \mathrm{C}$ until needed.

Before use the samples were sonicated briefly to clarify the total homogenate solution. A Bradford assay was performed to quantify the amount of total protein within each sample, using BSA as a standard. SDS-PAGE sample buffer was added to each protein sample and boiled for $5 \mathrm{~min}$. Based on the results from the Bradford analysis, the protein samples were normalized, and equivalent amounts of protein were loaded and resolved on a $10 \%$ acrylamide Tris/Tricine gel. The protein was transferred onto Immobilon-P membrane (Millipore, Bedford, MA) via a semi-dry transfer apparatus (Millipore). The membrane was blocked overnight with 5\% BSA. The next day the membrane was incubated in TBST buffer and 2\% BSA with $0.5 \mu \mathrm{g} / \mathrm{ml}$ of Erk antibody. Immunoreactive bands were visualized by using an alkaline phosphatase-conjugated secondary antibody and the chemiluminescence kit CDP-star (New England Biolabs, Beverly, MA). X-ray film (Kodak XAR5, Rochester, NY) was exposed to the membrane for several minutes and developed for visualization of the immunoreactivity bands. Multiple exposures of each membrane were taken to ensure the linearity of the immunoreactive bands, which were quantified by using ImageQuant. Then the membranes were stripped by using two $10 \mathrm{~min}$ washes of $\beta$-mercaptoethanol-containing stripping buffer $(62 \mathrm{~mm}$ Tris- $\mathrm{HCl}, \mathrm{pH}$ $6.8,2 \%$ SDS, and $100 \mathrm{~mm} \beta$-mercaptoethanol) at $60^{\circ} \mathrm{C}$. The stripped membranes were washed thoroughly in TBST, and immunoblotting was performed again with an antibody to phospho-Erk.
Histology. At the end of all behavioral experiments the rats were killed by $\mathrm{CO}_{2}$ overdose, followed by decapitation. The brains were removed and postfixed by using the protocol described in Immunohistochemistry. Brain sections $40 \mu \mathrm{m}$ thick were prepared with a cryostat and mounted on slides for verification of injection sites at $20 \times$ magnification.

Statistics. A one-way ANOVA test versus control was used for statistical comparison of cell counts in control and experimental tissue preparations and for hippocampal Western blots. A Student's $t$ test for paired variables was used for statistical comparison between the Western immunoblotting data taken from medial prefrontal punches after trace and after the unpaired control. Additionally, a $t$ test was used to compare the percentage of contextual freezing between vehicle and U0126-infused groups. A two-way repeated measures ANOVA test was used for statistical evaluation of the percentage of freezing to the CS between vehicle and U0126-infused groups. A $p$ value of $<0.05$ was used as the criterion for statistical significance. Raw data were used for all statistical analyses.

\section{Results}

\section{Hippocampal trace conditioning} memory storage

In humans, trace conditioning is impaired by hippocampal damage (McGlincheyBerroth et al., 1997; Clark and Squire, 1998). Animal post-training lesion studies have shown that the hippocampus is involved in memory storage and/or expression of trace fear conditioning (McEchron et al., 1998; Quinn et al., 2002). We, therefore, tested whether the hippocampus stores memory for trace fear conditioning by examining Erk activity in this structure after training. Figure $1 A$ shows representative Western blots for phospho- and total Erk at various time points after trace conditioning or after CS-alone presentations. Similar to previous reports that used conditioned emotional response (Atkins et al., 1998), trace fear conditioning significantly increased Erk phosphorylation $1 \mathrm{hr}$ after training (CS-alone, $n=4$; trace, $1 \mathrm{hr}$, $n=6 ; p<0.05$ ) (Fig. $1 B$ ). This increase in phosphorylation was not a result of an increase in total Erk levels. Erk phosphorylation had returned to control levels by $4 \mathrm{hr}$ after training.

To determine whether long-term memory storage for trace conditioning requires the hippocampus, animals were given eight CS-US pairings with a $20 \mathrm{sec}$ trace period and a pseudorandom intertrial interval (ITI) of 1-4 min (Fig. 1C). At $15 \mathrm{~min}$ after the completion of training, bilateral intrahippocampal infusions of either $1 \mu \mathrm{g}$ of U0126 (an inhibitor of MAP/Erk kinase, MEK) or vehicle were performed. Previous investigations have shown that U0126 is a selective inhibitor for the Erk cascade and that it does not block the activity of several other kinases, including calcium/calmodulin-dependent kinase II (CaMKII), protein kinase C (PKC), cAMP-dependent protein kinase (PKA), stressactivated protein kinase (SAPK), and P38 mitogen-activated protein kinase (MAPK) (Roberson et al., 1999; Davies et al., 2000). Long-term memory was assessed by monitoring freezing behavior during the trace period following tone presentation at 48 and $72 \mathrm{hr}$ after training. The percentage of time the animals remained frozen at each testing time point was averaged for four tone presentations. Figure $1 D$ shows that animals infused with U0126 had 
an impairment in the retention of CS-US association as indicated by decreased freezing behavior to the tone ( $-\mathrm{U} 0126, n=9$; $+\mathrm{U} 0126, n=8$; $p<0.05$ ). Additionally, post-training intrahippocampal U0126 infusion resulted in impaired retention of context-US association as indicated by decreased freezing behavior in the training context $(p<0.05$; Fig. $1 E)$.

\section{Trace conditioning and mPFC Erk phosphorylation}

To determine whether the mPFC can store long-term memory, we examined the phosphorylation of Erk in this structure. Western blot analysis of prelimbic/infralimbic punches $\left(\sim 1 \mathrm{~mm}^{3}\right)$ taken immediately after trace fear conditioning or immediately after an equal number of unpaired presentations of the CS and US was performed. Figure $2 A$ shows representative Western blots indicting an increase in the phosphorylation, but not total levels, of Erk in the prelimbic/infralimbic cortices as a result of trace conditioning. The summary results presented in Figure $2 B$ show that trace fear conditioning significantly increases Erk phosphorylation as compared with the unpaired controls (unpaired, $n=5$; trace, immediate, $n=7 ; p<0.05)$. This increase in Erk phosphorylation was not observed at the 1 or $4 \mathrm{hr}$ time points after trace conditioning (data not shown).

To examine further whether prefrontal Erk phosphorylation is specific to trace conditioning, we performed immunohistochemistry for phospho-Erk at varying time points after trace conditioning and compared them with a series of controls. Figure $2 C$ shows representative photomicrographs of the prelimbic area of the mPFC. Consistent with our Western blot results, the figure shows that there is an apparent increase in the number of phospho-Erk-positive cells immediately after trace conditioning. Additionally, an increase in Erk phosphorylation was observed in the infralimbic cortex, but not in the adjacent primary motor cortex (results not shown). To quantify the number of phosphoErk-positive cells, we used an unbiased stereological counting methodology as described in Materials and Methods. Figure $3 \mathrm{~A}$ shows an illustration of a coronal section through the prefrontal cortex, with the region defined for cell counting indicated by a black box. A significant increase in the number of phospho-Erkpositive cells per unit volume $\left(2.15 \times 10^{-2} \mathrm{~mm}^{3}\right)$ was observed immediately after trace fear conditioning $(n=6)$ relative to CSalone $(n=6)$, US-alone $(n=4)$, delay conditioning $(n=4)$, and unpaired CS-US $(n=4)$ control groups $(p<0.05$; Fig. $3 B)$. When individual cortical layers (visualized by using the neuronal marker NeuN) were assessed, increases in the number of phospho-Erk-positive cells were observed in layers II, III, and V-VI (data not shown). Prelimbic Erk phosphorylation had returned to control levels by $1 \mathrm{hr}$ after training $(n=4)$. To determine whether the phospho-Erk immunoreactivity observed in the prelimbic cortex was neuron-specific, we double-labeled tissue sections with phospho-Erk and Rip, an oligodendrocyte marker, GFAP, an astroglial marker, or NeuN, a neuronal marker (Fig. 3C). Only NeuN was observed to colocalize with phospho-Erk.

\section{Role of mPFC in memory storage}

As was the case in the hippocampus, the training-related increase in $\mathrm{mPFC}$ phospho-Erk immunoreactivity we observed after trace conditioning suggests an involvement of this structure in longterm memory storage. To examine this, we performed intra-prelimbic/infralimbic infusions of U0126. To determine the extent of Erk inhibition after intra-prelimbic/infralimbic U0126 infusion $(1 \mu \mathrm{g})$, we performed phospho-Erk immunohistochemistry. Figure $4 A$ shows that U0126 infusion into the mPFC resulted in a
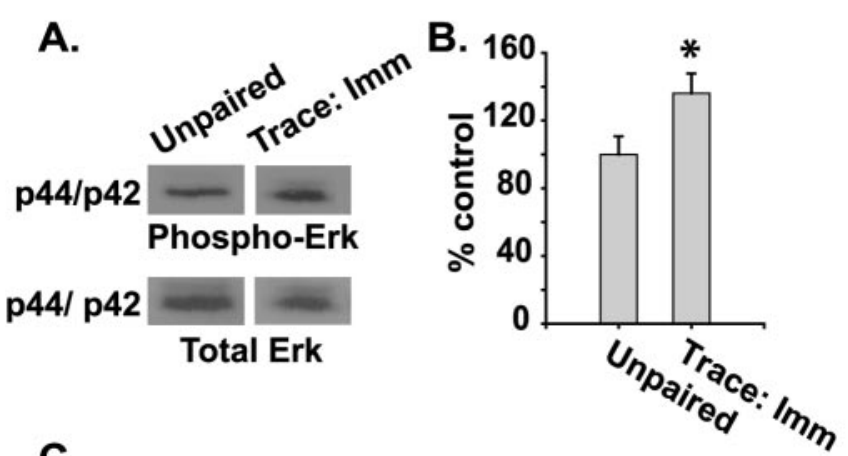

C.

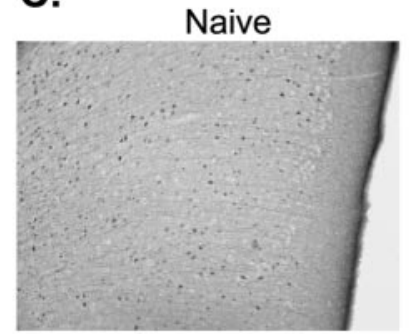

US Alone

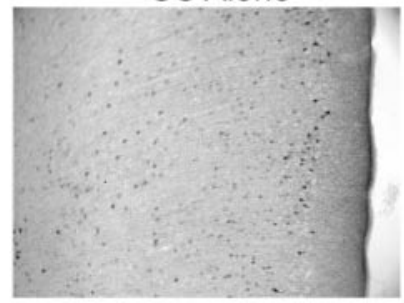

Unpaired

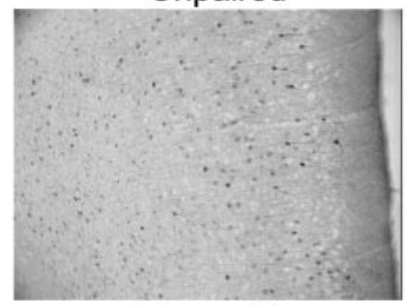

Trace: $1 \mathrm{hr}$
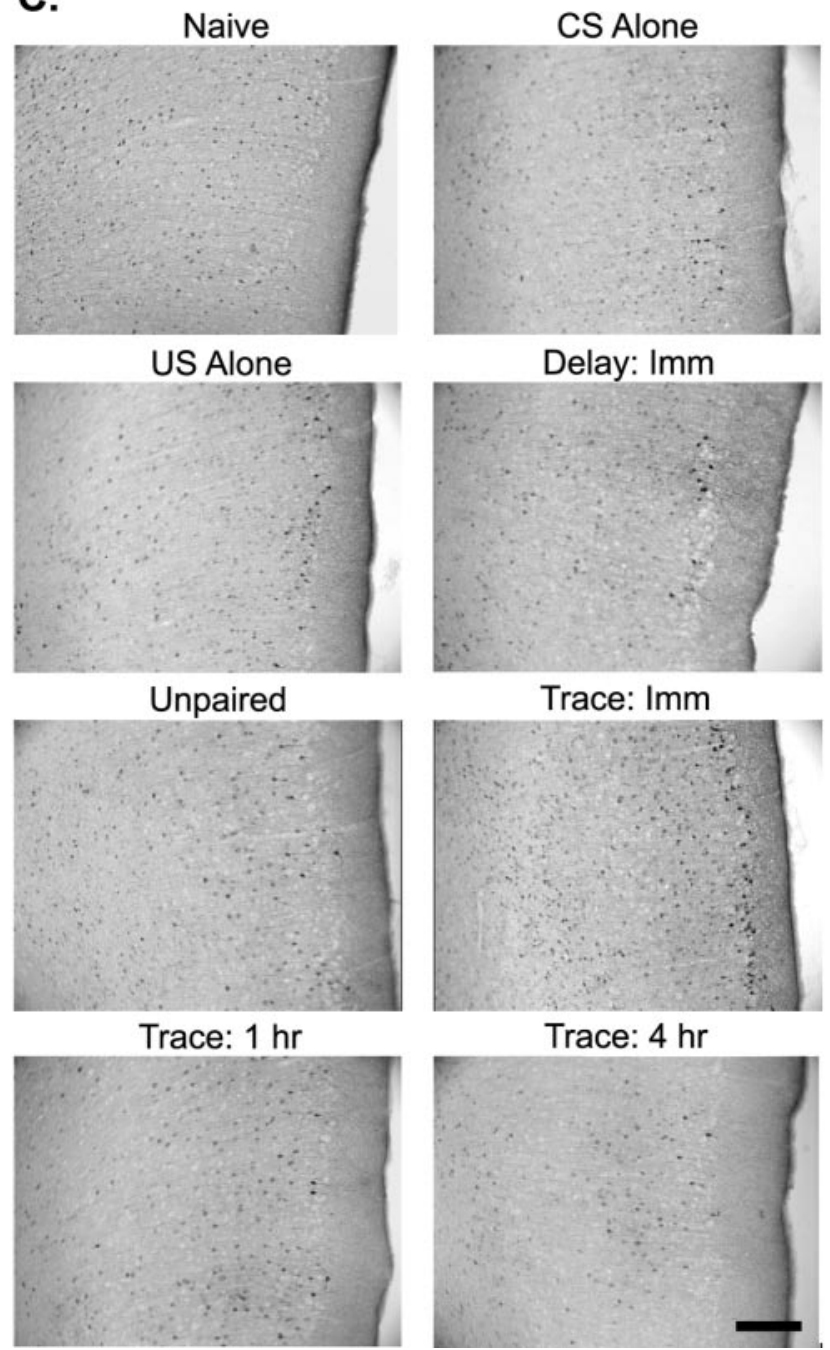

Delay: Imm

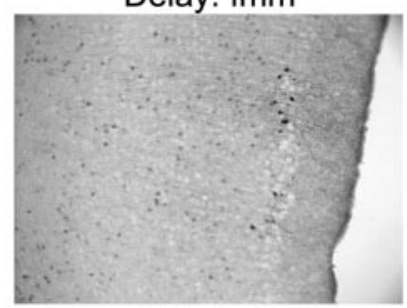

Trace: Imm

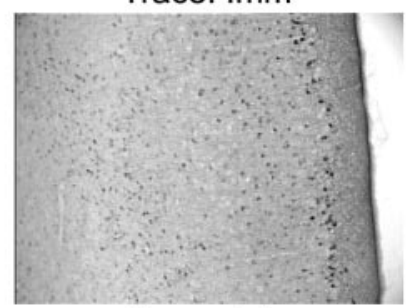

Trace: $4 \mathrm{hr}$

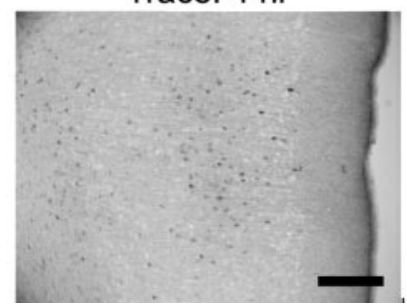

Figure 2. Increases in MPFC Erk phosphorylation correlate with the association of stimuli separated by time. A, Representative Western blots for dually phosphorylated Erk (p44/42) and total Erk in samples taken from the prelimbic/infralimbic cortices. $B$, Graph displays the mean \pm SEM phospho-Erk immunoreactivity of prefrontal samples taken immediately after each condition, normalized by using the total Erk immunoreactivity of the same blots. C, Photomicrographs of immunohistochemistry for dually phosphorylated Erk in the prelimbic/infralimbic cortices in naive, CS-alone, US-alone, delay conditioning, unpaired, and immediately and 1 and $4 \mathrm{hr}$ after trace fear conditioning. The figure shows an increase in Erk-positive cells specifically after trace conditioning. Scale bar, $200 \mu \mathrm{m}$. The asterisk indicates a significant difference as compared with control.

marked reduction in mPFC phospho-Erk immunoreactivity as compared with the vehicle-infused contralateral hemisphere. No reduction of phospho-Erk immunoreactivity was observed in adjacent regions such as the primary motor cortex or the cortical 
A.

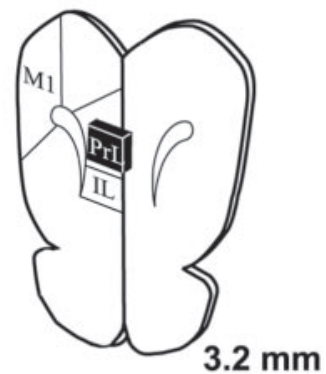

C.
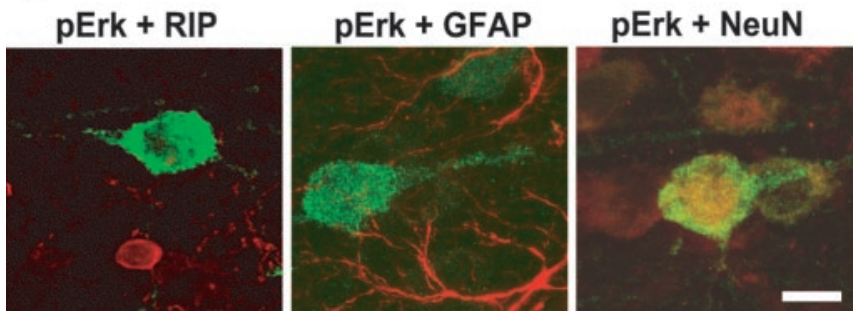

Figure 3. Trace conditioning increases phospho-Erk-positive neurons in the prelimbic cortex. $A$, Illustration of a coronal section through the medial prefrontal cortex $(+3.2 \mathrm{~mm}$ to bregma). The prelimbic region (black box) was defined for stereological cell counts. IL, Infralimbic; M1, primary motor cortex (adapted from Paxinos and Watson, 1986). B, Graph of the mean \pm SEM of phospho-Erk-positive cells counted per unit volume $\left(2.15 \times 10^{-2} \mathrm{~mm}^{3}\right)$ after each behavioral procedure. C, Representative confocal immunohistochemistry images of $\mathrm{mPFC}$ tissue sections prepared after trace fear conditioning, demonstrating that phospho-Erk (green) is not colocalized with Rip (red), a marker for oligodendrocytes, or with GFAP (red), a marker for astroglia, but is colocalized with NeuN (red), a marker for neurons, within the prelimbic cortex. Scale bar, $10 \mu \mathrm{m}$. The asterisk indicates a significant difference as compared with control.

regions surrounding the anterior olfactory nucleus (data not shown). Additionally, phospho-CaMKII in the $\mathrm{MPFC}$ was unaffected by U0126 infusion (Fig. $4 \mathrm{~B}$ ), suggesting that the reduction of phospho-Erk was not attributable to a general decrease in the immunoreactivity of the tissue. Because hippocampal Erk phosphorylation is required for long-term memory for trace conditioning, the influence of intra-prelimbic/infralimbic U0126 infusion on hippocampal Erk phosphorylation was assessed. No effect of intra-prelimbic/infralimbic U0126 administration was observed on hippocampal Erk phosphorylation (Fig. 4C).

Memory retention in the mPFC was examined by bilateral intra-prelimbic/infralimbic infusion of $1 \mu \mathrm{g}$ of U0126 or vehicle 10 min before training in the trace conditioning task (Fig. 5A). No significant difference in trace conditioning acquisition was observed between the U0126-infused $(n=11)$ and vehicleinfused animals $(n=10$; Fig. $5 B)$. When tested for memory retention at 48 and $72 \mathrm{hr}$ after training, the U0126-infused animals showed a significant decrease in freezing response as compared with the vehicle-infused controls $(p<0.05$; Fig. $5 B)$. Although not statistically significant $(p=0.054)$, a trend toward increased freezing was observed in the U0126-treated group when reexposed to the training context in the absence of the CS (Fig. 5C). To limit any interference in the learning of trace fear conditioning that could have occurred from pretraining U0126 infusions but that may have been undetectable by behavioral analysis, we performed immediate post-training $1 \mu \mathrm{g}$ U0126 infusions (Fig. $5 A$ ). Figure $5 D$ shows that long-term retention also was impaired when Erk was inhibited immediately after training (-U0126, $n=$ 12 ; $+\mathrm{U} 0126, n=12 ; p<0.05)$. Interestingly, when the animals were tested for contextual fear, a significant increase in freezing
Control
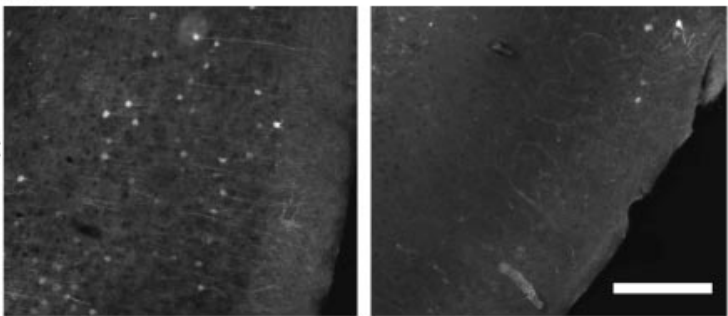

B.
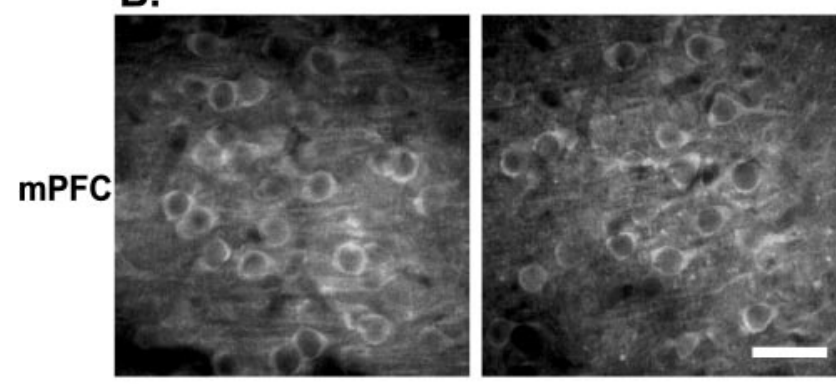

C.

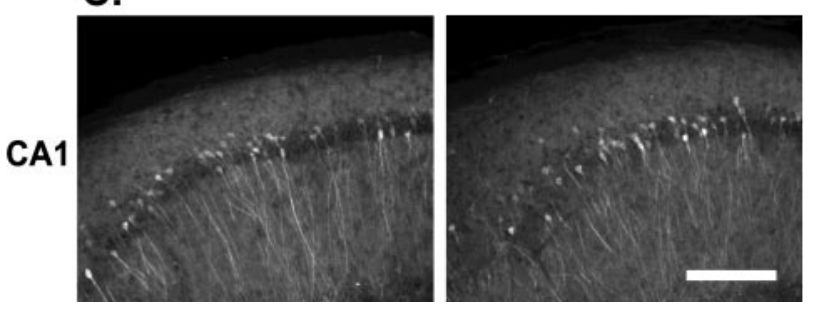

Figure 4. Intra-prelimbic/infralimbic U0126 infusion inhibits trace conditioning-related phospho-Erk in the $\mathrm{MPFC}$, but not in the hippocampus. $A$, Photomicrographs illustrating that pretraining intra-prelimbic/infralimbic U0126 infusion decreases prelimbic cortical Erk phosphorylation. Scale bar, $200 \mu \mathrm{m}$. B, Photomicrographs showing that intra-prelimbic/infralimbic U0126 infusion had no effect on prelimbic cortical CaMKII phosphorylation relative to vehicleinfused hemispheres. Scale bar, $30 \mu \mathrm{m}$. C, Additionally, intra-prelimbic/infralimbic U0126 infusion had no effect on hippocampal CA1 Erk phosphorylation relative to vehicle-infused hemispheres after trace fear conditioning. Scale bar, $200 \mu \mathrm{m}$.

behavior was observed in the U0126-treated group as compared with the vehicle-treated controls $(p<0.05$; Fig. $5 E)$.

To ensure that the differences in retention observed $48 \mathrm{hr}$ after drug infusion were not caused by a general mPFC dysfunction, we examined the ability of the animals to acquire and remember the trace conditioning task at this post-infusion time point. Animals were trained and tested for their ability to acquire the trace conditioning task (Fig. 6A). All rats were able to acquire the task as indicated by increased freezing behavior during the trace periods (data not shown). The animals 1 week later were divided into two comparable groups, and intra-prelimbic/infralimbic infusions of either vehicle $(n=8)$ or U0126 $(n=8)$ were performed. At $48 \mathrm{hr}$ after the infusion the animals were retrained in the trace conditioning task. This paradigm was used to test whether prefrontal function was altered as a result of mPFC U0126 infusions performed $48 \mathrm{hr}$ earlier, mimicking the conditions experienced during the trace conditioning testing (Fig. 5). No significant effect of U0126 pre-infusion was observed on retraining acquisition or retention (Fig. $6 B, C$ ), indicating that the impairment observed in Figure 5 was attributable to a disruption of memory storage rather than a generalized $\mathrm{mPFC}$ dysfunction. After the completion of behavioral studies the animals were killed, and brain sections were examined for cannula placement. Figure $6 D$ 
A.

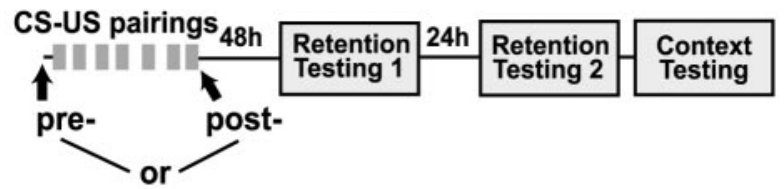

Pre-training infusion

B.

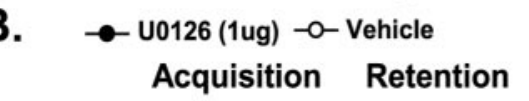

C.

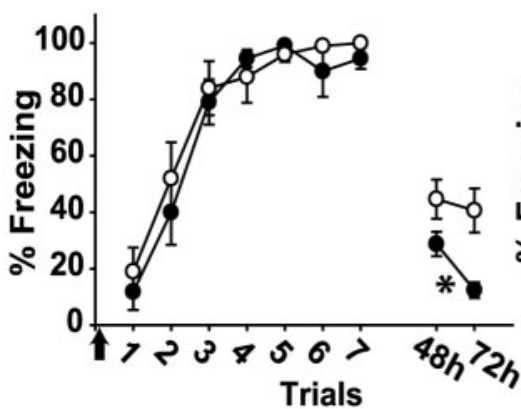

Context test

Post-training infusion

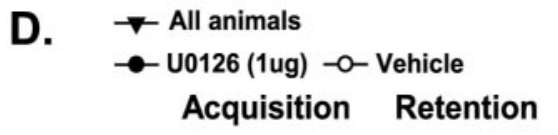

E.
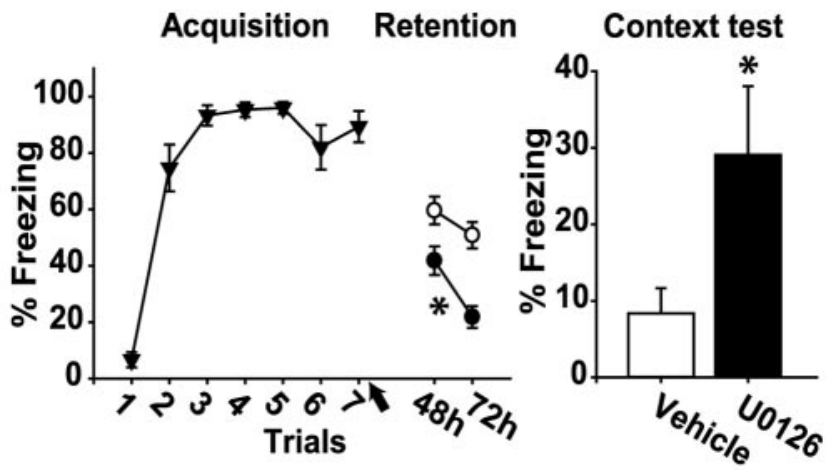

Figure 5. Pre- and post-training inhibition of Erk activity in the prelimbic/infralimbic cortex impairs long-term memory for trace fear conditioning. $A$, Illustration of the sequence of pretraining or post-training intra-prelimbic/infralimbic infusions, trace-conditioning training trials (CS-US pairings), and retention testing. $B$, Graph displaying the mean \pm SEM freezing behavior expressed as a percentage during acquisition and CS-US retention testing for animals receiving pretraining intra-prelimbic/infralimbic U0126 infusion versus vehicle. C, Graph displaying the mean \pm SEM freezing behavior expressed as a percentage during context-US retention testing for animals receiving pretraining intra-prelimbic/infralimbic U0126 infusion versus vehicle. $D$, Graph displaying the mean \pm SEM freezing behavior expressed as a percentage during acquisition and CS-US retention testing for animals receiving post-training intraprelimbic/infralimbic U0126 infusion versus vehicle. $E$, Graph displaying the mean \pm SEM freezing behavior expressed as a percentage during context-US retention testing for animals receiving post-training intra-prelimbic/infralimbic U0126 infusion versus vehicle. Asterisks indicate a significant difference as compared with control.

shows a photomicrograph of a representative infusion track (arrow). The summary in Figure $6 D$ displays the infusion sites of a representative sample group.

\section{Discussion}

Previous studies have demonstrated a role for the prefrontal cortex in the association of multimodal stimuli separated by time (Fuster et al., 2000). The results presented in this report extend the role of the prefrontal cortex to include the storage of memory for these associations. The present study demonstrates two key

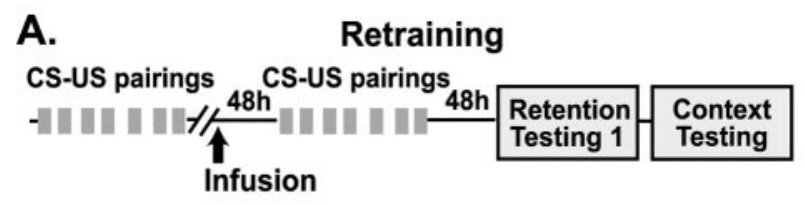

B. $\rightarrow$ U0126 (1ug) -0 -Vehicle C.

Acquisition Retention Context test

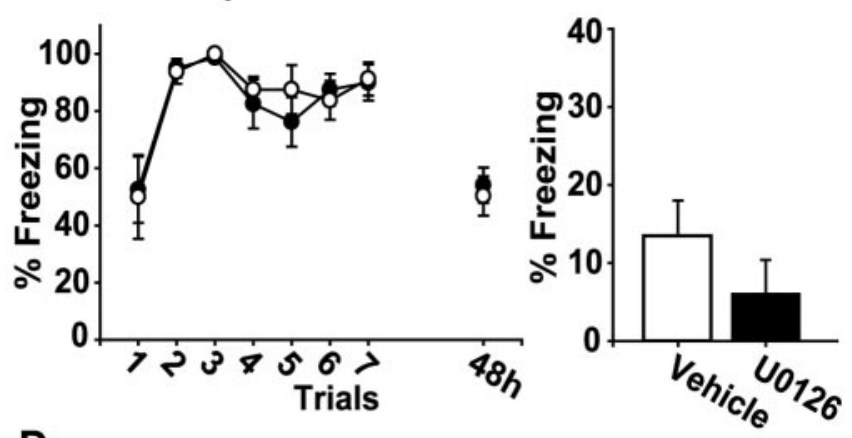

D.

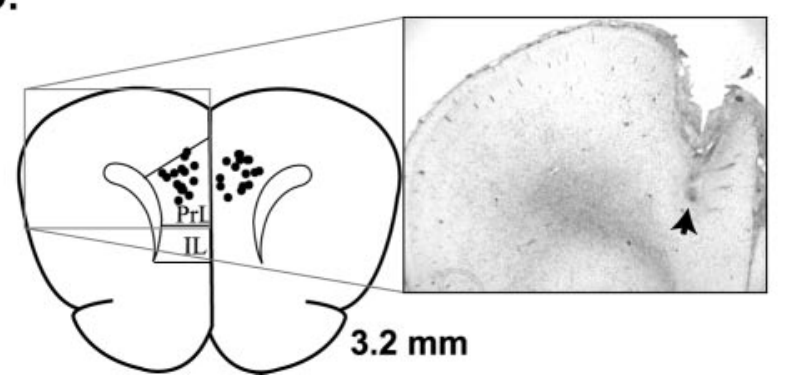

Figure 6. Trace conditioning retention deficits are not a result of a general dysfunction of $\mathrm{mPFC}$. A, Illustration of the sequence of trace-conditioning training (CS-US pairings), intraprefrontal infusion, retraining trials, and retention testing. $B$, Graph displaying the mean \pm SEM freezing behavior expressed as a percentage during acquisition and CS-US retention for animals receiving intra-prelimbic/infralimbic U0126 infusions performed $48 \mathrm{hr}$ before retraining versus vehicle. C, Graph displaying the mean \pm SEM freezing behavior expressed as a percentage during context-US retention testing for animals receiving post-training intra-prelimbic/infralimbic U0126 infusions performed $48 \mathrm{hr}$ before retraining versus vehicle. D, Simplified illustration of a coronal section of a rat brain, $+3.2 \mathrm{~mm}$ anterior to bregma (adapted from Paxinos and Watson, 1986), displaying the sites of intra-prelimbic/prelimbic infusion from a representative sample group of behaviorally tested animals. The photomicrograph shows the guide and infusion tracks from a representative tissue section. The arrow indicates the terminus of the track.

findings: (1) mPFC stores long-term memory for trace fear conditioning, a multi-modal, cross-temporal associative task; and (2) prefrontal memory storage may be required for establishing the relevancy of task-related cues. To the best of our knowledge, these attributes have not been ascribed previously to the prefrontal cortex.

Trace CS-US associative memory

In previous reports, long-term memory storage has been correlated to the activation of Erk within specific brain structures (Berman et al., 1998; Blum et al., 1999; Schafe et al., 2000; Hebert and Dash, 2002). This is consistent with our results indicating a requirement for Erk activation in the hippocampus for long-term memory of trace fear conditioning. Additionally, as detected by Western blot analysis and immunohistochemistry, trace fear conditioning increased Erk phosphorylation within mPFC neurons immediately after training. However, no increase in Erk phosphorylation was observed in the primary motor cortex, sug- 
gesting that trace conditioning did not induce an indiscriminate increase in Erk phosphorylation. Furthermore, our findings suggest that the increase in mPFC Erk activation that was observed is specific to associative memory between stimuli or events separated by time, because no change in Erk phosphorylation was observed after unpaired or delay conditioning paradigms. These correlative results advocate a role for the $\mathrm{mPFC}$ in trace conditioning memory storage.

Consistent with previous studies showing short-term memory (Martin et al., 1997; Schafe et al., 2000) and acquisition (Blum et al., 1999) to be unaffected by Erk inhibition, pretraining Erk inhibition in the $\mathrm{MPFC}$ had no effect on trace acquisition. However, blockade of Erk activity in the mPFC before or immediately after training impaired memory when it was tested 48 and $72 \mathrm{hr}$ later. Control experiments demonstrated that inhibition of mPFC Erk activity $48 \mathrm{hr}$ before training had no effect on the animals' ability to acquire or retain trace conditioning, which has been shown previously to require prefrontal function (Kronforst-Collins and Disterhoft, 1998; Weible et al., 2000; McLaughlin et al., 2002). These findings, taken with the observation that intra-prefrontal infusions of U0126 had no effect on CaMKII $\mathrm{Thr}^{286}$ autophosphorylation (Giese et al., 1998) after training, suggest that mPFC Erk inhibition-induced deficits in trace conditioning retention result from a disruption of memory storage processes and not from a generalized mPFC dysfunction.

Examination of the time course for Erk activation in the mPFC shows that Erk phosphorylation was enhanced immediately after the completion of training and returned to control levels by $1 \mathrm{hr}$. Conversely, a significant increase in Erk phosphorylation was detected in the hippocampus $1 \mathrm{hr}$, but not immediately, after training. This later time course for hippocampal Erk activation after trace conditioning is similar to that observed in the hippocampus after a conditioned emotional response task (Atkins et al., 1998). Additionally, Erk activity after the completion of training was required in both the prefrontal cortex and the hippocampus for long-term CS-US associative memory. Because Erk activation is a correlate for long-term memory formation, this suggests that long-term memory-related signal transduction has a similar time course, if not an earlier time course, in the $\mathrm{mPFC}$ as in the hippocampus. It has been postulated that, for hippocampal-dependent tasks, memory storage occurs first in the hippocampus and over time is transferred to cortical areas (for review, see Squire et al., 1984; McClelland et al., 1995). However, our data show that aspects of a hippocampal-dependent memory are stored in the mPFC before, or at least parallel to, hippocampal memory storage, presumably as a direct result of learning. This finding questions the validity of current memory consolidation models.

\section{Memory for relevancy}

Our behavioral experiments also demonstrate that inhibition of $\mathrm{mPFC}$ Erk signaling resulted in an increase in contextual freezing $72 \mathrm{hr}$ after training in a task designed to undermine context-US association and promote CS-US association. Lesion studies (Winocur and Olds, 1978; Kim and Fanselow, 1992) and systemic administration of an Erk cascade inhibitor (Atkins et al., 1998) have been used to suggest hippocampal plasticity in contextual memory storage. Here, we demonstrate by direct intrahippocampal infusion of an Erk cascade inhibitor that contextual associative memory is stored in the hippocampus. Although a hippocampal-dependent contextual association is made during the training, our data suggest that the $\mathrm{MPFC}$ may be storing information relative to its pertinence. Therefore, when memory storage was disrupted in the mPFC, the memory that the context was not an accurate or relevant predictor of US onset was impaired, giving rise to a significant increase in contextual fear. An alternative explanation for the observed increase in contextual fear may be that, in the absence of CS-US associative memory, context-US association is strengthened. However, based on our experimental data and on previous reports, this second explanation is unlikely. For example, at the time of contextual testing, CS-US association had been extinguished in both vehicle and U0126-infused animals. Further, although a decrease in the retention of CS-US association was observed after intrahippocampal U0126 infusion, a decrease rather than an increase in contextual freezing was observed, suggesting that the mPFC is involved specifically in storing memory about the relevancy of formed associations.

The role of prefrontal function in establishing task relevancy has been well documented. In humans, imaging data have shown prefrontal activity to be involved in distinguishing between taskrelevant and nonrelevant information represented by posterior cortical regions (Potts and Tucker, 2001; Staines et al., 2002). Additionally, in primates single-cell activity within the prefrontal cortex has been observed to respond differently to task-relevant cues than to task-irrelevant cues (Yajeya et al., 1988). In rats, prefrontal neural activity has been observed to discriminate the relevancy of variables in an odor discrimination task, including the assigned reward contingency of a current odor (Schoenbaum and Eichenbaum, 1995). The distinction between relevant and irrelevant cues and the importance of these cues in predicting outcome engage prefrontal function in humans, nonhuman primates, and in the rat. Consistent with this role for the prefrontal cortex, our data suggest that the $\mathrm{mPFC}$ stores information related to the relevancy of past experience in predicting probable outcome from external cues and that this information is used in directing behavioral response.

\section{Prefrontal memory function}

In humans, functional imaging studies have demonstrated that the prefrontal cortex is active during both episodic memory encoding and retrieval (Shallice et al., 1994; Tulving et al., 1994). Further, frontal lobe damage has been reported to impair markedly the recall of episodic and autobiographical memories (Incisa della Rocchetta and Milner, 1993; Calabrese et al., 1996; Rousseaux et al., 1998). These and other studies clearly have delineated a function for the prefrontal cortex, not only during the encoding but in the retrieval of memory. However, lesion and imaging experiments are unable to answer whether prefrontal cortex directly participates in memory storage. The results presented here demonstrate that long-term memory storage takes place in the $\mathrm{mPFC}$ in rats, more or less, at the same time as in the hippocampus. The information stored within the prefrontal cortex may contribute to functions already attributed to this structure, such as forming and recalling meaningful associations (Dolan and Fletcher, 1997), as well as directing appropriate behavioral response (Harlow, 1848; Shallice, 1988; Lauria, 1996; Morris et al., 1997). Prefrontal memory may be needed for directing retrieval by distinguishing relevant from nonrelevant associations.

\section{References}

Atkins CM, Selcher JC, Petraitis JJ, Trzaskos JM, Sweatt JD (1998) The MAPK cascade is required for mammalian associative learning. Nat Neurosci 1:602-609.

Berman DE, Hazvi S, Rosenblum K, Seger R, Dudai Y (1998) Specific and differential activation of mitogen-activated protein kinase cascades by 
unfamiliar taste in the insular cortex of the behaving rat. J Neurosci 18:10037-10044.

Blum S, Moore AN, Adams F, Dash PK (1999) A mitogen-activated protein kinase cascade in the CA1/CA2 subfield of the dorsal hippocampus is essential for long-term spatial memory. J Neurosci 19:3535-3544.

Calabrese P, Markowitsch HJ, Durwen HF, Widlitzek H, Haupts M, Holinka B, Gehlen W (1996) Right temporofrontal cortex as critical locus for the ecphory of old episodic memories. J Neurol Neurosurg Psychiatry 61:304-310.

Clark RE, Squire LR (1998) Classical conditioning and brain systems: the role of awareness. Science 280:77-81.

Coggeshall RE, Lekan HA (1996) Methods for determining numbers of cells and synapses: a case for more uniform standards of review. J Comp Neurol 364:6-15.

Crow T, Xue-Bain JJ, Siddiqi V, Neary JT (1998) Phosphorylation of mitogen-activated protein kinase by one-trial and multi-trial classical conditioning. J Neurosci 18:3480-3487.

Dash PK, Mach SA, Blum S, Moore AN (2002) Intrahippocampal wortmannin infusion enhances long-term spatial and contextual memories. Learn Mem 9:167-177.

Davies SP, Reddy H, Caivano M, Cohen P (2000) Specificity and mechanism of action of some commonly used protein kinase inhibitors. J Biochem 351:95-105.

Dias R, Robbins TW, Roberts AC (1996) Dissociation in prefrontal cortex of affective and attentional shifts. Nature 380:69-72.

Dias R, Robbins TW, Roberts AC (1997) Dissociable forms of inhibitory control within prefrontal cortex with an analog of the Wisconsin card sort test: restriction to novel situations and independence from "on-line" processing. J Neurosci 17:9285-9297.

Dolan RJ, Fletcher PC (1997) Dissociating prefrontal and hippocampal function in episodic memory encoding. Nature 388:582-585.

Everling S, Tinsely CJ, Gaffan D, Duncan J (2002) Filtering of neural signals by focusing attention in the monkey prefrontal cortex. Nat Neurosci 5:671-676.

Fuster JM, Alexander GE (1971) Neuron activity related to short-term memory. Science 173:652-654.

Fuster JM, Bodner M, Kroger J (2000) Cross-modal and cross-temporal association in neurons of frontal cortex. Nature 405:347-351.

Giese KP, Fedorov NB, Filipkowski RK, Silva AJ (1998) Autophosphorylation at $\mathrm{Thr}^{286}$ of the alpha calcium-calmodulin kinase II in LTP and learning. Science 279:870-873.

Harlow, JM (1848) Passage of an iron rod through the head. Boston Med Surg J 39:389-393.

Hebert AE, Dash PK (2002) Extracellular signal-regulated kinase activity in the entorhinal cortex is necessary for long-term spatial memory. Learn Mem 9:156-166.

Incisa della Rocchetta A, Milner B (1993) Strategic search and retrieval inhibition: the role of the frontal lobe. Neuropsychologia 31:503-524.

Kim JJ, Fanselow MS (1992) Modality-specific retrograde amnesia of fear. Science 256:675-677.

Kronforst-Collins MA, Disterhoft JF (1998) Lesions of the caudal area of rabbit medial prefrontal cortex impair trace eyeblink conditioning. Neurobiol Learn Mem 69:147-162.

Lauria AR (1996) Higher cortical functions in man. New York: Basic Books.

Markowitsch HJ, Calabrese P, Liess J, Haupts M, Durwen HF, Gehlen W (1993) Retrograde amnesia after traumatic injury of the fronto-temporal cortex. J Neurol Neurosurg Psychiatry 56:988-992.

Martin KC, Michael D, Rose JC, Barad M, Casadio A, Zhu H, Kandel ER (1997) MAP kinase translocates into the nucleus of the presynaptic cell and is required for long-term facilitation in the Aplysia. Neuron 18:899-912.

McClelland JL, McNaughton BL, O'Reilly RC (1995) Why there are complementary learning systems in the hippocampus and neocortex: insights from the successes and failures of connectionist models of learning and memory. Psychol Rev 102:419-457.

McEchron MD, Bouwmeester H, Tseng W, Weiss C, Disterhoft JF (1998)
Hippocampectomy disrupts auditory trace conditioning and contextual fear conditioning in the rat. Hippocampus 8:638-646.

McGlinchey-Berroth R, Carrillo MC, Gabrieli JDE, Brawn CM, Disterhoft JF (1997) Impaired trace eyeblink conditioning in bilateral, medialtemporal lobe amnesia. Behav Neurosci 111:873-882.

McLaughlin J, Skaggs H, Churchwell J, Powell DA (2002) Medial prefrontal cortex and Pavlovian conditioning: trace versus delay conditioning. Behav Neurosci 116:37-47.

Morris RG, Miotto EC, Feigenbaum JD, Bullock P, Polkey CE (1997) The effect of goal-subgoal conflict on planning ability after frontal- and temporal-lobe lesions in humans. Neuropsychologia 35:1147-1157.

Pavlov IP (1927) Conditioned reflexes: an investigation of the physiological activity of the cerebral cortex. London: Oxford UP.

Paxinos G, Watson C (1986) The rat brain in stereotaxic coordinates. New York: Academic.

Potts GF, Tucker DM (2001) Frontal evaluation and posterior representation in target detection. Brain Res Cogn Brain Res 11:147-156.

Quinn JJ, Oommen SS, Morrison GE, Fanselow MS (2002) Post-training excitotoxic lesions of the dorsal hippocampus attenuate forward trace, backward trace, and delay fear conditioning in a temporally specific manner. Hippocampus 12:495-504.

Roberson ED, English JD, Adams JP, Selcher JC, Kondratick C, Sweatt JD (1999) The mitogen-activated protein kinase cascade couples PKA and $\mathrm{PKC}$ to camp response element binding protein phosphorylation in area CA1 of hippocampus. J Neurosci 19:4337-4348.

Rousseaux M, Godefroy O, Cabaret M (1998) Learning disorders after ruptured aneurysms of the anterior communicating artery. Rev Neurol (Paris) 154:508-522.

Schafe GE, Atkins CM, Swank MW, Bauer EP, Sweatt JD, LeDoux JE (2000) Activation of ERK/MAP kinase in the amygdala is required for memory consolidation of Pavlovian fear conditioning. J Neurosci 20:8177-8187.

Schoenbaum G, Eichenbaum H (1995) Information coding in the rodent prefrontal cortex. II. Ensemble activity in orbitofrontal cortex. J Neurophysiol 74:751-761.

Schoenbaum G, Chiba AA, Gallagher M (1998) Orbitofrontal cortex and basolateral amygdala encode expected outcomes during learning. Nat Neurosci 1:155-159.

Shallice T (1988) From neuropsychology to mental structure. New York: Cambridge UP.

Shallice T, Fletcher P, Frith CD, Grasby P, Frackowiak RS, Dolan RJ (1994) Brain regions associated with acquisition and retrieval of verbal episodic memory. Nature 368:633-635.

Squire LR, Cohen NJ, Nadel L (1984) The medial temporal region and memory consolidation: a new hypothesis. In: Memory consolidation (Weingartner H, Parker E, eds), pp 185-210. Hillsdale, NJ: Erlbaum.

Staines WR, Graham SJ, Black SE, Mcllroy WE (2002) Task-relevant modulation of contralateral and ipsilateral primary somatosensory cortex and the role of a prefrontal-cortical sensory gating system. NeuroImage 15:190-199.

Tulving E, Kapur S, Markowitsch HJ, Craik FI, Habib R, Houle S (1994) Neuroanatomical correlates of retrieval in episodic memory: auditory sentence recognition. Proc Natl Acad Sci USA 91:2012-2015.

Vann SD, Brown MW, Erichsen JT, Aggleton JP (2000) Fos imaging reveals differential patterns of hippocampal and parahippocampal subfield activation in rats in response to different spatial memory tests. J Neurosci 20:8144-8152.

Weible AP, McEchron MD, Disterhoft JF (2000) Cortical involvement in acquisition and extinction of trace eyeblink conditioning. Behav Neurosci 114:1058-1067.

Winocur G, Olds J (1978) Effects of contextual manipulation on memory and reversal learning in rats with hippocampal lesions. J Comp Physiol Psychol 92:312-321.

Yajeya J, Quintana J, Fuster JM (1988) Prefrontal representation of stimulus attributes during delay tasks. II. The role of behavioral significance. Brain Res 6:222-230. 